\title{
Daily fluctuations in occupation with and worry about COVID-19
}

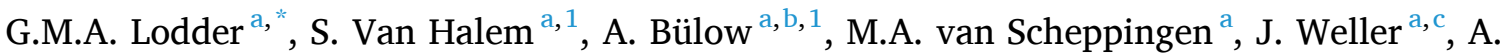 \\ K. Reitz ${ }^{\mathrm{a}}$ \\ ${ }^{a}$ Department of Developmental Psychology, Tilburg University, the Netherlands \\ ${ }^{\mathrm{b}}$ Department of Psychology, Education and Child Studies, Erasmus University of Rotterdam, the Netherlands \\ ${ }^{\mathrm{c}}$ Centre for Decision Research, University of Leeds Business School, United Kingdom
}

\section{A R T I C L E I N F O}

\section{Keywords:}

COVID-19

Worry

Preoccupation

Dynamic relationships

Within-person effects

Neuroticism

\begin{abstract}
A B S T R A C T
In the first week after the first COVID-19 patient was reported in the Netherlands, we conducted a pre-registered momentary assessment study (7 surveys per day, 50 participants, 7 days) to study the dynamic relationship between individuals' occupation with and worries about COVID-19 in daily life, and the moderating role of neuroticism in this relationship. At the group level, higher scores on occupation and worry co-occurred, and occupation predicted worry $1 \mathrm{~h}$ later, but not vice versa. There were substantial individual differences in the magnitudes and directions of the effects. For instance, occupation with COVID-19 was related to increases in worry for some but decreases in worry for others. Neuroticism did not predict any of these individual differences in the links between worry and occupation. This study suggests that it is important to go beyond group-level analyses and to account for individual differences in responses to COVID-19.
\end{abstract}

\section{Introduction}

\subsection{Daily fluctuations in occupation with and worry about COVID-19}

Starting in early 2020, news of the rapid spread of COVID-19, a rising death toll, overwhelmed health systems, and major changes in individuals' daily life, occupied the thoughts of billions of people worldwide. Since the outbreak, there were clear increases in anxiety and worry related to COVID-19 (Ahorsu et al., 2020; Gao et al., 2020; Qiu et al., 2020). This widespread increase in worry is reason for concern, because repeated occurrences of daily worries might be the foundation of long-term psychological (mal)adjustment, such as the development of depression and anxiety disorders (Hoyer et al., 2001). Understanding the daily dynamics of worries is also needed to identify coping strategies to prevent psychological (mal)adjustment (Hong, 2007).

One key factor that might contribute to worry about COVID-19 is the degree to which individuals are occupied with (information about) COVID-19 on a daily basis. Constant news updates likely impact the degree to which people are occupied with COVID-19, both in terms of their behavior (i.e., talking about and looking up information about COVID-19) and their thoughts. The World Health Organization advised people to reduce their news intake to avoid worry from constant exposure to stressful news (WHO, 2020). Indeed, studies reported positive associations between (social) media exposure and COVID-19 related anxiety (Gao et al., 2020; Garfin et al., 2020; Mertens et al., 2020).

To date, the studies about occupation with COVID-19 and worry are mostly cross-sectional, but the relationship between worry and occupation with COVID-19 is likely a within-person process that unfolds over time, possibly on a daily or even hourly level. The goal of the present study was to examine the temporal dynamics between worry about and occupation with COVID-19 using the Experience Sampling Method (ESM; see Bolger et al., 2003; Larson \& Csikszentmihalyi, 2014). Moreover, we examined whether neuroticism may explain some of the individual differences in the momentary relationships between worry about and occupation with COVID-19. The 21st century brings manifold new challenges (the pandemic being one, but also climate change, technological changes and so forth) and hence, uncertainty that people need to deal with. Understanding how different people respond to such stressors, is important for prevention and intervention programs (e.g. from a resilience perspective). We measured occupation with and worry about COVID-19 multiple times per day. The advantages of such a design include the ecological validity, reduced risk of recall bias (Myin-Germeys et al., 2009), and the ability to study the direction of an effect and

\footnotetext{
* Corresponding author at: Developmental Psychology, Tilburg University, Warandelaan 2, 5037 AB Tilburg, the Netherlands.

E-mail address: g.m.a.lodder@uvt.nl (G.M.A. Lodder).

1 These authors made equal contributions and thus share second authorship.
} 
the heterogeneous experiences that individuals may have. That is, looking up information on COVID-19 might increase worry (cf. Mertens et al., 2020), but can also be a consequence of worrying.

The data for the present study were collected during a critical time of the outbreak in the Netherlands: the week after February 25, when the first COVID-19 patient was reported in The Netherlands. During this week, the potential risk of COVID-19 became clear to the public, making it an especially relevant week to examine predictors of worry regarding this emergent risk. Moreover, during this week, the Dutch government took various measures to limit the spread of COVID-19 for the first time (e.g., the advice to work from home, for details, see Appendix A). We examined the relationship between worry about and occupation with COVID-19 in a student sample, which is an interesting group as the college years are a sensitive developmental period, and increases in mental health problems during COVID-19 seem especially prevalent in young adults (Bu et al., 2020).

\subsection{Momentary relations between worry about and occupation with COVID-19}

We expect that people differ in the association between their worry about and occupation with COVID-19. Worry may function to prioritize the potential hazard of a risk and activate coping strategies, which helps increase perceived control, for instance by taking preventative actions to reduce the risk (Peters et al., 2006). Thus, worry may lead to increases in occupation with COVID-19, which for some people could be an adaptive process, as staying informed about a potential risk factor can help people exert control over their situation, which can consequently reduce their initial levels of worry (Griffin et al., 1999). Thus, worry may increase occupation with COVID-19 for some. For others, worry and subsequent occupation with COVID-19 may lead to a cycle that maintains, and perhaps even increases worry (Chae, 2015). As media coverage can increase anxiety and worry about emergent disease (Kasperson et al., 1988), being occupied with COVID-19 may facilitate repetitive negative thinking, which can maintain emotional problems (Ehring \& Watkins, 2008). Some people may therefore choose to avoid occupation with COVID-19 when they experience worry. Thus, we hypothesize that for some people, occupation with COVID-19 is associated with decreases in worry in the next moment, and for others to increases (H1.1, for an overview of hypotheses, see Fig. 1). Similarly, we hypothesize that worry about COVID-19 relates to increases in occupation with COVID-19 the next moment for some, but to decreases in worry for others (H1.2).

\subsection{Moment-to-moment variability of worry about COVID-19}

Besides the effect of occupation with COVID-19 on worry and vice versa, individuals can differ in the extent to which worry in one moment predicts worry in the next moment. Emotional inertia refers to the degree to which emotional states are resistant to change, when mood is carried from one situation to the next (Kuppens et al., 2007). For some people, worry about COVID-19 may also spill over from one situation to the next, which might pose a risk for long-term psychological maladjustment (Kuppens et al., 2012), whereas for others worry might wane faster (i.e., we expect significant between-person differences in the within-person autoregressive effect of worry, H1.3). We have no directional hypotheses about the general (average) effect of individuals' tendency to remain occupied with COVID-19 (i.e., about the autoregressive effect of occupation), but we will explore whether there is a general tendency at a group level.

\subsection{The role of neuroticism in worrying about COVID-19}

Between-person differences, such as differences in personality, partly explain why people respond differently to experiences in daily life (Geukes et al., 2017). Specifically, neuroticism may explain individual differences in the associations between worry about and occupation with COVID-19. Neuroticism is related to greater intolerance of uncertainty (Lauriola et al., 2016), and to greater negative affect on days when negative events occur (see Lahey, 2009 for an overview). In turn, negative mood is related to looking up information when there is no apparent problem (Albarracin \& Hart, 2011; Das \& Fennis, 2008). Thus, highly neurotic individuals may actively search for, or focus on, negative information. We expect that neuroticism is related to a stronger positive effect of occupation with COVID-19 to worry about COVID-19 from one moment to the next (H2.1), and that average level of worry about COVID-19 is higher for individuals who score higher on neuroticism (H2.2). All hypotheses and the plan of analyses for the present study were preregistered (see https://osf.io/wvhp2/).

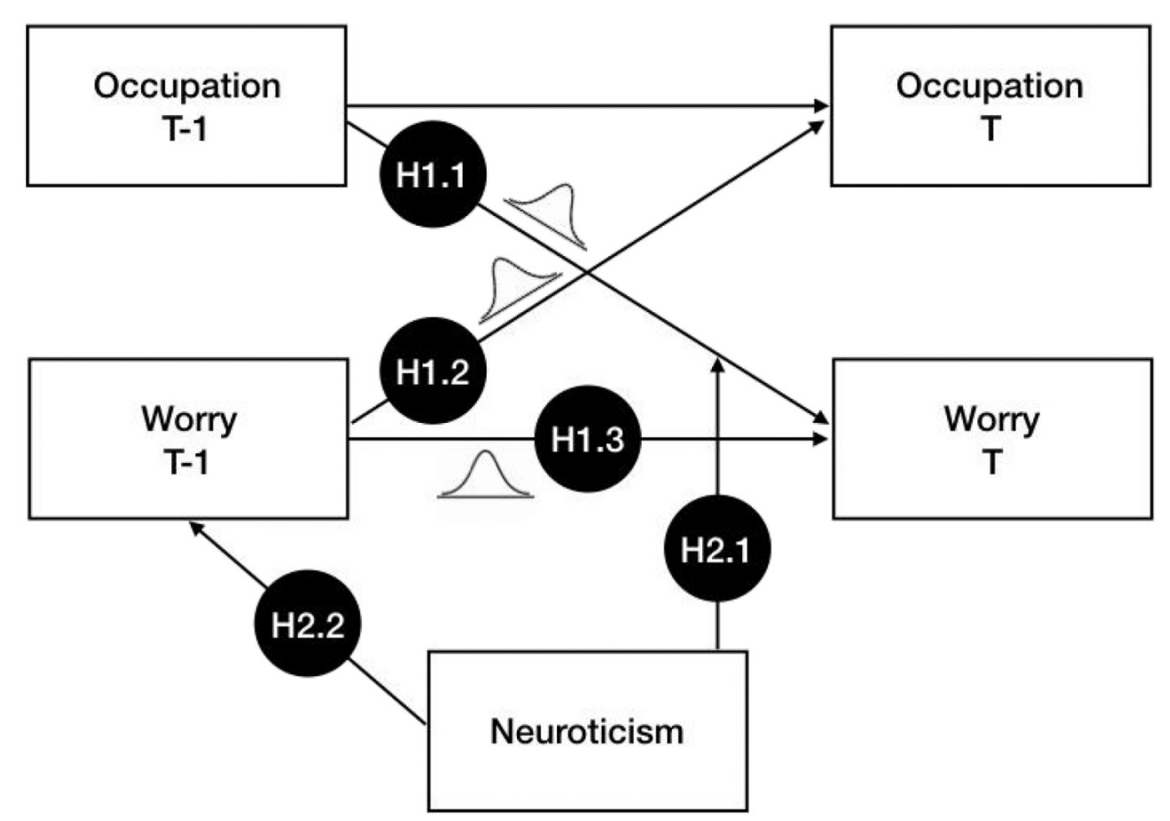

Fig. 1. Research framework depicting the hypotheses in a cross-lagged model of occupation with COVID-19 and worry about COVID-19 for one interval (T-1 to T1). 


\section{Methods}

\subsection{Procedure}

The study consisted of a baseline questionnaire (completed between February 26, 2020 and March 1, 2020) and 1 week of 7 momentary assessments per day (March 2, 2020-March 8, 2020). The study was originally designed as a student project to examine the effect of contraception on affect and differences in affective processes between Dutch and international students. The day after the baseline measure was completed, the first case of COVID-19 in The Netherlands was reported. We then added 4 questions about occupation with and worry about COVID-19 to the ESM study. After the ESM study, we collected data in both the existing sample and in a control sample for validation (see Appendix F). Our initial study and amendments were approved by the Ethics Review Board (EC-2020.RP47) of Tilburg University, The Netherlands. All participants provided active consent for participation. Participants were recruited through the university's research participation system. Psychology students are obliged to take part in research and receive course credit for their participation. Students self-enroll in the research of their choosing. They received course credit for completing the baseline questionnaire and at least $75 \%$ of the momentary questionnaires. We used a smartphone application (Ethica data) to collect the momentary data across a period of seven days, starting on Monday March 2. For details, see Appendix B.

\subsection{Participants}

Given the original goal of the study, the sample included females only, with an equal distribution of Dutch and international students. Our sample consisted of 50 1st year female students of Psychology. The number of participants was set because of the feasibility of the students' project, and was pre-registered (see https://osf.io/wvhp2/). On average, participants completed 39.32 momentary questionnaires per person, bringing the total number of measurements to 1966, which is sufficient to run DSEM models and common in ESM studies (van Roekel et al., 2019). Of the 50 participants, 27 (54\%) were Dutch and 23 (46\%) were international ( 14 German students, and 9 students from other locations in Europe, Asia and South America). Their age ranged between 18 and $25\left(M_{\text {age }}=19.74, S D_{\text {age }}=1.68\right)$.

\subsection{Materials}

\subsubsection{Momentary measures}

2.3.1.1. Worry about COVID-19. To measure participants' momentary worry about COVID-19, we asked them to rate "How worried do you feel about the coronavirus right now" on a VAS scale ranging from (0) "not at all" to (100) "extremely".

2.3.1.2. Occupation with COVID-19. To measure participant's momentary occupation with COVID-19 we asked: "Please indicate how much time you spend since the last beep doing the following" (...) 1) "Looking up information about the coronavirus", 2) "talking about the coronavirus", and 3) "thinking about the coronavirus". Answers were administered using a VAS scale ranging from (0) "not at all" to (100) "a lot". Both within- and between-person reliability of the scale was good $\left(\omega_{\mathrm{W}}=0.78\right.$; $\left.\omega_{\mathrm{B}}=0.92\right)$. Multilevel confirmatory factor analysis indicated a satisfactory fit $\left(X^{2}(3)=25.48, p<.001\right.$, RMSEA $=0.06$, CFI $=0.94$, TLI $=$ 0.83 ). Factor loadings ranged between 0.58 and 0.90 for the withinperson level and between 0.80 and 0.97 for the between-person level (for inter-item correlations, see Table 1).

2.3.1.3. Positive and negative affect. Affect was used in some sensitivity analyses and was measured with 12 items that were administered at all
Table 1

Inter-item correlations between the items of the occupation with COVID-19 Scale.

\begin{tabular}{llllll}
\hline & 1 & 2 & 3 & \multicolumn{1}{l}{$M$} & \multicolumn{1}{l}{$S D$} \\
\hline 1. Thinking about COVID-19 & - & 0.89 & 0.74 & 12.78 & 19.47 \\
2. Talking to others about COVID-19 & 0.63 & - & 0.75 & 12.71 & 20.53 \\
3. Looking up information about & 0.52 & 0.40 & -1 & 7.58 & 16.53 \\
$\quad$ COVID-19 & & & & & \\
\hline
\end{tabular}

Note. Correlations above the diagonal represent within-person correlations, correlations below the diagonal represent between-person correlations.

momentary assessments. The items were selected to match the circumplex model of affect (Russell, 1980), and included items such as "I feel happy" and "I feel agitated". Answers were administered using a VAS scale ranging from (0) "disagree" to (100) "agree".

\subsubsection{Baseline measure}

2.3.2.1. Neuroticism. Neuroticism was measured using the Big Five Inventory-2 (BFI-2; Soto \& John, 2017). Participants rated 12 items in the neuroticism domain scale on a 5-point scale ranging from 1 (disagree strongly) to 5 (agree strongly). A sample item is "I am someone who is moody, has up and down mood swings". The reliability for this scale was $\operatorname{good}(\alpha=0.90)$.

\subsection{Analytical strategy}

Before analyzing the data, we checked for random answer patterns by using items of the Positive and Negative Affect Scale (PANAS; Watson et al., 2007). We expected the items within both affect scales to correlate for each person, and found that for all individuals, correlations of the items within a scale were higher than $r=0.10$, therefore, no participants were excluded from the main analysis.

We used a Multilevel Vector Autoregressive Model (ML-VAR; Schuurman et al., 2016), using a Dynamic Structural Equation Modelling framework (DSEM; Asparouhov et al., 2017) in Mplus (Muthén \& Muthén, 2017). See https://osf.io/wvhp2/?view_only=ef02ae3f71a64 $586 \mathrm{ba} 36 \mathrm{e} 1 \mathrm{dc} 436 \mathrm{f} 25 \mathrm{a} 5$ for a detailed pre-registered plan of analyses and code, and appendix B for all used materials. The ML-VAR Model estimates the lagged and concurrent within-person associations of worry about COVID-19 and occupation with COVID-19. Furthermore, at the between-person level, we investigated between-person differences in the random slopes, as well as the correlation between the random slopes, random intercepts and the between-person predictor neuroticism. Given the relatively small sample size for between-person analyses and the novelty of the research area, we used an alpha level of 0.05 to determine significance in all analyses.

The tinterval option was used to consider unequally spaced measurements, as the questionnaires were distributed at random time points during pre-defined intervals. The model with a pre-registered time interval of $2 \mathrm{~h}$ the model did not converge. Therefore, we adjusted this to $1 \mathrm{~h}$. This model converged and resulted in $25 \%$ present data, which is above the acceptable minimum of $10 \%$ present data for VAR models (Asparouhov et al., 2017). Therefore, all lagged effects can be interpreted as the changes that occur within $1 \mathrm{~h}$.

\subsection{Data statement}

All data and scripts are available at https://osf.io/wvhp2/

\section{Results}

\subsection{Descriptive statistics}

Table 1 shows the descriptive statistics (for more details, see 
Appendix G). The distributions of the scores of worry about and occupation with COVID-19 were right-skewed, indicating that despite the turbulent time during which our data collection took place, most participants did not report experiencing being highly worried about, or occupied with, COVID-19. The variance between individuals in neuroticism (see Table 2) spanned almost the entire scale, ranging from 1.42 to 4.42 on a 5-point scale, suggesting that our sample included both participants who were relatively emotionally stable and those that were relatively neurotic. The nomological network of variables indicated positive relationships between occupation and worry both on the within-person $(r=0.43)$ and between-person $(r=0.76)$ level, but no significant relationship between neuroticism being worried $(r=-0.09)$ or occupied with COVID-19 $(r=0.05)$.

\subsection{Fixed within-person associations between worry and occupation about COVID-19}

Table 3 displays the fixed within-person associations of the VAR model, that is, the average relationship between our measures within moments, within individuals, accounting for all other parameters. In line with the correlations reported in Section 3.1, a positive within-person correlation indicated that occupation and worry within the same measurement occasion co-occurred. The stability paths indicate that in general, when people were occupied with or worried about COVID-19 at one time point, they also tended to be occupied with or worried about COVID-19 at the next time point. Finally, the within-person cross-lagged paths suggest that being occupied with COVID-19 in a particular moment predicted worrying about COVID-19 at the next moment. However, on average, being worried at one time point was not significantly related to being occupied with COVID-19 at the next time point.

\subsection{Individual differences in within-person associations}

Fig. 2 displays the time series of the relationship between occupation and worry across the study for two participants (for a complete overview of all time-series, see Appendix C). This illustrates how diverse the relationship between occupation and worry can be: For some participants, worry and occupation seemed to go hand in hand (left panel), whereas for other participants, they seemed to be unrelated (right panel).

Table 3 displays the average (pooled) within-person associations. We found significant variances around the fixed within-person parameters (beta's) for both stability paths (Worry-Worry $\sigma^{2}=0.29, p<.001$; Occupation-Occupation $\sigma^{2}=0.26, p<.001$ ) and both cross-lagged paths (Worry-Occupation $\sigma^{2}=0.310, p<.001$; Occupation-Worry $\sigma^{2}=1.10$, $p<.001)$. Thus, for all stability paths and cross-lagged effects there were relevant individual differences in the magnitude of these parameters.

Fig. 3 depicts the distribution of individual parameters (Betas) of these relationships. For all parameters, for many participants the betas were close to zero. This indicates that in our sample, despite the average within-person effects reported in Table 3, for many people the relationships that we tested were actually weak or barely present. However, in line with our hypotheses, for all paths there was substantial variability between individuals. The top left panel shows the variation in

Table 2

Descriptive statistics of experience sampling items across measurements and participants.

\begin{tabular}{lllllll}
\hline & Mean (SD) & Min-max & Skewness & Kurtosis & N & ICC \\
\hline Worry & $\begin{array}{l}17.52 \\
\text { (21.95) }\end{array}$ & $0-100$ & 1.59 & 4.69 & 1966 & 0.21 \\
Occupation & $\begin{array}{l}11.02 \\
(16.30)\end{array}$ & $0-100$ & 2.21 & 8.26 & 1967 & 0.44 \\
Neuroticism & $3.09(0.76)$ & $1.42-4.42$ & -0.46 & 0.34 & 50 & - \\
\hline
\end{tabular}

Note. ICC = Intra Class Correlation coefficient.
Table 3

Within-person fixed effects of the relationships between occupation with and worry about COVID-19.

\begin{tabular}{|c|c|c|c|c|}
\hline & \multirow[t]{2}{*}{ Estimate $^{\mathrm{a}}$} & \multirow{2}{*}{$\begin{array}{l}p- \\
\text { Value }^{\mathrm{b}}\end{array}$} & \multicolumn{2}{|c|}{$95 \%$ confidence interva } \\
\hline & & & $\begin{array}{l}\text { Lower } \\
2.5 \%\end{array}$ & $\begin{array}{l}\text { Upper } \\
2.5 \%\end{array}$ \\
\hline \multicolumn{5}{|l|}{ Within-person correlation } \\
\hline Occupation \& worry & 0.34 & $<.001$ & 0.29 & 0.40 \\
\hline \multicolumn{5}{|l|}{ Within-person stability paths } \\
\hline Worry $\mathrm{T} 1$ - worry $\mathrm{T} 2$ & 0.15 & $<.001$ & 0.08 & 0.21 \\
\hline $\begin{array}{l}\text { Occupation } \mathrm{T} 1 \text { - occupation } \\
\text { T2 }\end{array}$ & 0.24 & $<.001$ & 0.17 & 0.31 \\
\hline \multicolumn{5}{|l|}{ Within-person cross-lagged paths } \\
\hline Worry $\mathrm{T} 1$ - occupation $\mathrm{T} 2$ & -0.01 & .447 & -0.08 & 0.21 \\
\hline Occupation $\mathrm{T} 1$ - worry $\mathrm{T} 2$ & 0.14 & $<.001$ & 0.07 & 0.20 \\
\hline
\end{tabular}

${ }^{\text {a }}$ Estimates are standardized using the STDYX Standardization (within-level standardized estimates averaged over clusters).

b Bayesian equivalent to one-tailed $p$-values.

the stability paths of worry which indicates that some people seemed to be caught in worry (i.e., once they were worried, they stayed worried), but for others, there were more fluctuations in worry. Importantly, as the average levels of worry in this sample were modest, strong autoregressive effects could also indicate the tendency to stay unworried about COVID-19. The bottom-right panel depicts the stability paths of occupation with COVID-19. We had no a-priori hypothesis about the autoregressive effect of occupation, but this effect showed a similar pattern to worry.

The cross-lagged effects in the bottom-left and top-right panels of Fig. 3 indicate that for some people, occupation with COVID-19 was related to less worry at the next time point (negative betas), indicating a relief effect for thinking about, talking about and looking up information about COVID-19. For other people, however, occupation with COVID-19 was related to more worry at the next time point (positive betas), indicating a self-maintaining worrying effect. Similarly, some people seemed to become more occupied with COVID-19 after they experienced worry (positive betas), whereas other people seemed to become less occupied with COVID-19 when they were worried (negative betas).

\subsection{Associations with personality}

We examined whether neuroticism explained some of the significant individual differences in the within-person associations between worry and occupation about COVID-19. Table 4 displays correlations between the observed within-person random slopes ( $\Phi W W, \Phi O O, \Phi W O$ and $\Phi O W)$, random intercepts (worry and occupation) and neuroticism. Contrary to our hypotheses, none of the correlations with neuroticism reached significance (ranging between $r=-0.15$ and $r=0.13$, all $p$ 's $>$ .23; see Appendix G for scatterplots of these associations).

\subsection{Planned exploratory analyses}

We performed several exploratory analyses, which are described in the appendixes. In summary, we found no associations between the within-person effects and other Big Five personality constructs (see Appendix D), we found individual differences in change in worry and occupation over the study period using growth curve models (see Appendix E), we found no differences in levels of occupation and risk related behaviors between our participants and a control sample (see Appendix F), and no significant association between momentary negative effect and worry (see Appendix H).

\section{Discussion}

We used momentary assessments in the week after the first COVID- 

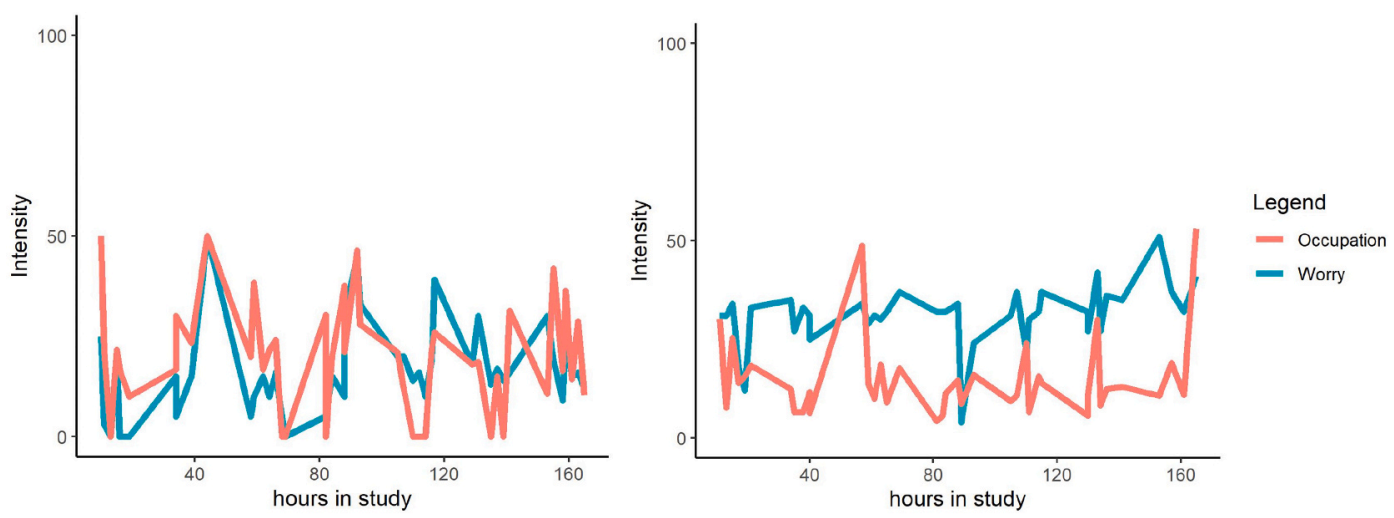

Fig. 2. Illustration of the fluctuations over time in worry about and occupation with COVID-19 of two participants (for a full overview of all participants, see Appendix C).
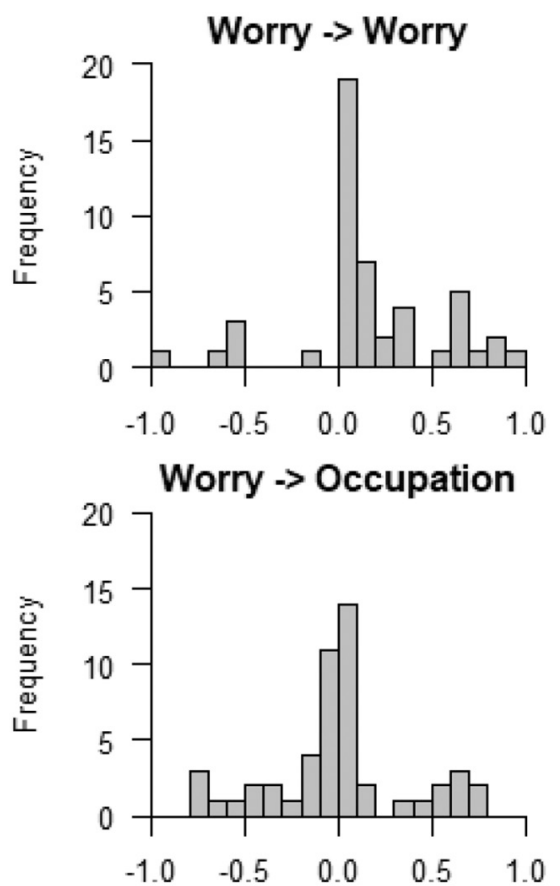
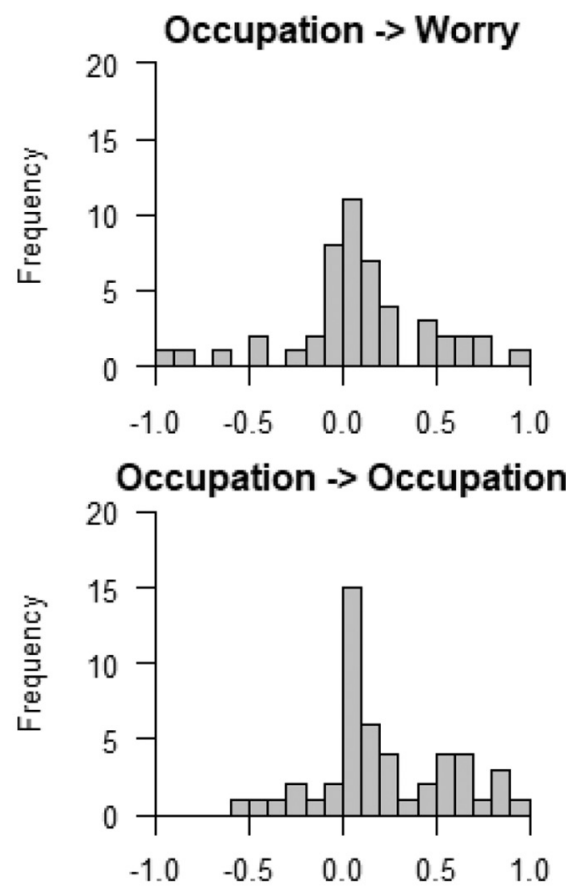

Fig. 3. Individual differences in within-person associations. Distribution of the individual parameters (Beta's) per person is displayed.

Table 4

Between-person correlations between effects in the VAR model and neuroticism.

\begin{tabular}{|c|c|c|c|c|c|c|c|}
\hline & $\Phi W W$ & $\Phi O O$ & ФWO & ФOW & Worry & Occup & Neuro \\
\hline$\Phi W W$ & 1 & & & & & & \\
\hline$\Phi О 0$ & $-0.49^{* *}$ & 1 & & & & & \\
\hline ФWO & $-0.52^{* *}$ & $0.66^{\text {***k }}$ & 1 & & & & \\
\hline Occup & -0.01 & $0.38^{*}$ & 0.16 & 0.15 & $0.83^{* * *}$ & 1 & \\
\hline Neuro & -0.07 & -0.15 & -0.02 & 0.13 & -0.10 & 0.03 & 1 \\
\hline
\end{tabular}

Note. $\Phi \mathrm{WW}=$ stability path worry, $\Phi \mathrm{OO}=$ stability path occupation, $\Phi \mathrm{WO}=$ cross-lagged path occupation T1 to worry $\mathrm{T} 2$, $\Phi \mathrm{OW}=$ cross-lagged path worry T1 to occupation $\mathrm{T} 2$.

* Bayesian equivalent to one-tailed $p$-values $<.05$.

** Bayesian equivalent to one-tailed $p$-values $<.01$.

***** Bayesian equivalent to one-tailed $p$-values $<.001$.

19 patient was reported in the Netherlands to provide novel insights into the dynamic relationship between occupation with and worry about COVID-19 in daily life in female psychology students. In contrast with previous cross-sectional studies (Gao et al., 2020; Garfin et al., 2020;
Mertens et al., 2020), we examined the dynamic relationship between occupation and worrying about COVID-19 as they unfolded in daily life, estimated individual differences in the relationship between both constructs, and assessed the degree to which these individual differences 
were related to neuroticism.

We found that levels of occupation with and worry about COVID-19 co-occurred within the same measurement occasion. Across time, we found that on average, being occupied with COVID-19 in a particular moment predicted being worried about COVID-19 in a later moment, but there was no significant relationship between being worried in one moment and later occupation. Moving beyond these average effects, we found substantial individual differences in the magnitudes and directions of these effects. For many participants, being occupied with COVID-19 was not related to their level of worry about COVID-19, whereas for others occupation was related to either decreases or increases in worry. We found similar individual differences in the stability of worry. Contrary to our hypotheses, neuroticism was not significantly associated with any of these individual differences.

Although our results are in line with the previous studies that examined the relationship between an individual's occupation with COVID-19 (in terms of information seeking) and worry about COVID-19, they provide important additional insights and nuances. First, whereas the rationale of previous studies was that occupation with COVID-19 causes increases in worry and not vice versa, such directed effects cannot be determined via cross-sectional methods. With longitudinal design, we were able to provide further evidence for this notion, as on average we found no evidence that people attempt to exert control over worry about COVID-19 by being more occupied with COVID-19, for instance by looking up information about it.

Second, we found that the magnitude and even the direction of the effects in our model varied substantially between individuals. For many individuals worry and occupation were unrelated, and for some, being occupied with COVID-19 seemed to have a comforting effect. We advise scholars to interpret our findings and previous findings bearing these considerable individual differences in mind. Given the individual differences in the link between occupation with COVID-19 and worry, there is no straightforward one-size-fits all advice for how to deal with the overwhelming amount of news, conversations and thoughts about COVID-19.

Third, we found no significant relationship between neuroticism and any of these individual differences. The lack of significant findings for between-person differences could be due to insufficient power. We had 1966 measures in total, but these measures were clustered in 50 people. Although our sample size is common for studies employing an ESM design to study within-person associations (van Roekel et al., 2019), the sample may have been underpowered for between-person analyses. Moreover, the relatively small and homogenous sample limits the generalizability of the present study. A larger sample would also allow more advanced between-person analyses, such as 3-way interactions between background, personality and within-person dynamics. Yet, given the large variation in neuroticism across the entire range of the scale and given that plots gave no indication of trends in the data (see Appendix G), we suggest that neuroticism may indeed be unrelated to temporal dynamics in worry about and occupation with COVID-19. Alternatively, we speculate that effects for neuroticism possibly only emerge after the initial adaptation to the new risk has completed.

Finally, it is noteworthy that - even though the news almost exclusively reported on COVID-19 during the week of data collection - most participants did not report high levels of daily worries about or occupation with COVID-19. In light of the previous literature that clearly observed an increase in anxiety and worry in response to the COVID-19 outbreak (e.g., Ahorsu et al., 2020; Asmundson \& Taylor, 2020; Gao et al., 2020; Qiu et al., 2020), we would have expected the average worry about COVID-19 in our sample to be higher. A potential explanation of this finding could be that although people may indicate that they are worried about COVID-19 when they are asked to consciously reflect on this, these worries may not come to light so much at an hourly level (i.e., momentary worry). Another explanation lies in the sample selection (i.e., female psychology students in their early 20s). This young sample might have a lower risk perception than older samples who may be more likely to have existing medical conditions that put them at risk for developing a poor progression of symptoms. Another reason for the low worry levels might be the rather high SES of university students, as socio-economic disadvantage is a risk factor for worry about to COVID19 (Zhou \& Guo, 2021). Taken together, the homogeneity of the sample is a limitation of the present study. Although replication of the present study is not possible due to the unique timing of the study (during the first week of the COVID-19 outbreak in the Netherlands), conceptual replication for other emergent risks in the future may be possible, and could benefit from utilizing a larger sample.

Taken together, our study suggests that on average, when individuals are occupied with COVID-19 in a particular moment, they are more likely to be worried about COVID-19 at a later moment. Yet, we found substantial individual differences in the magnitude of the effects in our model. This implies that a one-size-fits-all solution for how to cope with negative thoughts or emotions surrounding COVID-19 may not exist, which could also hold for future crises that are characterized by emerging societal risk as they manifest. Moreover, seemingly logical advice, such as "try to limit your news-intake, otherwise you will only be more worried" (WHO, 2020), may therefore not hold for everyone. In our sample, most participants did not experience increased worry after occupation with COVID-19, in fact, for some occupation with COVID-19 even decreased worry. Future research could follow up on this study by using ideographic analyses, as these may give more clarity about the (differences in) within-person associations between occupation and worry. Based on the current findings, it is clear that attention for individual differences in how we deal with the psychological consequences of COVID-19 is warranted.

\section{Funding}

This research was supported by the Dutch Research Council [Gerine Lodder, 016.Veni.195.186, Loes Keijsers, 452-17-011]; European Union's Horizon 2020 - Research and Innovation Framework Programme [Anne Reitz, 846839].

\section{CRediT authorship contribution statement}

Gerine Lodder: Conceptualization, Writing - original draft (outline, results, discussion), Writing - Review and editing, Supervision, Project administration

Sjoerd van Halem: Conceptualization, Writing - original draft (methods), Methodology, Investigation, Data curation

Anne Bülow: Conceptualization, Methodology, Formal analyses (main analyses), Data curation, Writing - original draft (pre-registration), Visualisation

Manon van Scheppingen: Conceptualization, Formal analyses (posthoc analyses in Appendix 5).

Joshua Weller: Conceptualization, Writing - Review and editing, Methodology (risk-related measures Appendix 5)

Anne Reitz: Conceptualization, Writing - original draft (introduction).

\section{Declaration of competing interest}

None.

\section{Appendix A. Supplementary data}

Supplementary data to this article can be found online at https://doi. org/10.1016/j.paid.2021.111078.

\section{References}

Ahorsu, D. K., Lin, C. Y., Imani, V., Saffari, M., Griffiths, M. D., \& Pakpour, A. H. (2020). The Fear of COVID-19 Scale: Development and initial validation. International 
Journal of Mental Health and Addiction. https://doi.org/10.1007/s11469-020-002708.

Albarracin, D., \& Hart, W. (2011). Positive mood + action = negative mood + inaction: Effects of general action and inaction concepts on decisions and performance as a function of affect. Emotion, 11(4), 951-957. https://doi.org/10.1037/a0024130.

Asmundson, G. J. G., \& Taylor, S. (2020). Coronaphobia: Fear and the 2019-nCoV outbreak. Journal of Anxiety Disorders, 70, Article 102196. https://doi.org/10.1016/ j.janxdis.2020.102196.

Asparouhov, T., Hamaker, E. L., \& Muthén, B. (2017). Dynamic structural equation models (pp. 1-71). Retrieved from http://www.statmodel.com/download/DSEM.pdf (submitted for publication).

Bolger, N., Davis, A., \& Rafaeli, E. (2003). Diary methods: Capturing life as it is lived. Annual Review of Psychology, 54(1), 579-616. https://doi.org/10.1146/annurev. psych.54.101601.145030.

Bu, F., Steptoe, A., \& Fancourt, D. (2020). Loneliness during lockdown: Trajectories and predictors during the COVID-19 pandemic in 35,712 adults in the UK. Social Science \& Medicine, 8, 141-149. https://doi.org/10.1101/2020.05.29.20116657.

Chae, J. (2015). Online cancer information seeking increases cancer worry. Computers in Human Behavior, 52, 144-150. https://doi.org/10.1016/j.chb.2015.05.019.

Das, E., \& Fennis, B. M. (2008). In the mood to face the facts: When a positive mood promotes systematic processing of self-threatening information. Motivation and Emotion, 32(3), 221-230. https://doi.org/10.1007/s11031-008-9093-1.

Ehring, T., \& Watkins, E. R. (2008). Repetitive negative thinking as a transdiagnostic process. International Journal of Cognitive Therapy, 1(3), 192-205. https://doi.org/ 10.1521/ijct.2008.1.3.192.

Gao, J., Zheng, P., Jia, Y., Chen, H., Mao, Y., Chen, S., ... Dai, J. (2020). Mental health problems and social media exposure during COVID-19 outbreak. PLoS One, 15(4). https://doi.org/10.1371/journal.pone.0231924.

Garfin, D. R., Silver, R. C., \& Holman, E. A. (2020). The novel coronavirus (COVID-2019) outbreak: Amplification of public health consequences by media exposure. Health Psychology, 39, 355-357. https://doi.org/10.1037/hea0000875.

Geukes, K., Nestler, S., Hutteman, R., Küfner, A. C. P., \& Back, M. D. (2017). Trait personality and state variability: Predicting individual differences in within- and cross-context fluctuations in affect, self-evaluations, and behavior in everyday life. Journal of Research in Personality, 69, 124-138. https://doi.org/10.1016/j. jrp.2016.06.003.

Griffin, R. J., Dunwoody, S., \& Neuwirth, K. (1999). Proposed model of the relationship of risk information seeking and processing to the development of preventive behaviors. Environmental Research, 80(2 II). https://doi.org/10.1006/ enrs.1998.3940.

Hong, R. Y. (2007). Worry and rumination: Differential associations with anxious and depressive symptoms and coping behavior. Behaviour Research and Therapy, 45(2), 277-290. https://doi.org/10.1016/j.brat.2006.03.006.

Hoyer, J., Becker, E. S., \& Roth, W. T. (2001). Characteristics of worry in gad patients, social phobics, and controls. Depression and Anxiety, 13(2), 89-96. https://doi.org/ 10.1002/da.1021.

Kasperson, R. E., Renn, O., Slovic, P., Brown, H. S., Emel, J., Goble, R., ... Ratick, S. (1988). The social amplification of risk: A conceptual framework. Risk Analysis, 8(2), 177-187. https://doi.org/10.1111/j.1539-6924.1988.tb01168.x.

Kuppens, P., Sheeber, L. B., Yap, M. B. H., Whittle, S., Simmons, J. G., \& Allen, N. B. (2012). Emotional inertia prospectively predicts the onset of depressive disorder in adolescence. Emotion, 12(2), 283-289. https://doi.org/10.1037/a0025046.
Kuppens, P., Van Mechelen, I., Nezlek, J. B., Dossche, D., \& Timmermans, T. (2007). Individual differences in core affect variability and their relationship to personality and psychological adjustment. Emotion, 7(2), 262-274. https://doi.org/10.1037/ 1528-3542.7.2.262.

Lahey, B. B. (2009). Public health significance of neuroticism. American Psychologist, 64 (4), 241-256. https://doi.org/10.1037/a0015309.

Larson, R., \& Csikszentmihalyi, M. (2014). The experience sampling method. In Flow and the foundations of positive psychology: The collected works of Mihaly Csikszentmihalyi (pp. 21-34). https://doi.org/10.1007/978-94-017-9088-8_2.

Lauriola, M., Foschi, R., Mosca, O., \& Weller, J. (2016). Attitude toward ambiguity: Empirically robust factors in self-report personality scales. Assessment, 23(3), 353-373.

Mertens, G., Gerritsen, L., Duijndam, S., Salemink, E., \& Engelhard, I. M. (2020). Fear of the coronavirus (COVID-19): Predictors in an online study conducted in March 2020. Journal of Anxiety Disorders, 74. https://doi.org/10.1016/j.janxdis.2020.102258.

Muthén, L. K., \& Muthén, B. O. (2017). Mplus user's guide. Los Angeles, CA: Muthén \& Muthén.

Myin-Germeys, I., Oorschot, M., Collip, D., Lataster, J., Delespaul, P., \& Van Os, J. (2009). Experience sampling research in psychopathology: Opening the black box of daily life. Psychological Medicine, 39(9), 1533-1547. https://doi.org/10.1017/ S0033291708004947.

Peters, E., Slovic, P., Hibbard, J. H., \& Tusler, M. (2006). Why worry? Worry, risk perceptions, and willingness to act to reduce medical errors. Health Psychology, 25 (2), 144-152. https://doi.org/10.1037/0278-6133.25.2.144.

Qiu, J., Shen, B., Zhao, M., Wang, Z., Xie, B., \& Xu, Y. (2020). A nationwide survey of psychological distress among Chinese people in the COVID-19 epidemic: Implications and policy recommendations. General Psychiatry, 33, Article e100213. https://doi.org/10.1136/gpsych-2020-100213.

Russell, J. A. (1980). A circumplex model of affect. Social Psychology, 39(6), 1161-1178. https://doi.org/10.1037/h0077714.

Schuurman, N. K., Ferrer, E., de Boer-Sonnenschein, M., \& Hamaker, E. L. (2016). How to compare cross-lagged associations in a multilevel autoregressive model. Psychological Methods, 21(2), 206-221. https://doi.org/10.1037/met0000062.

Soto, C. J., \& John, O. P. (2017). The next Big Five Inventory (BFI-2): Developing and assessing a hierarchical model with 15 facets to enhance bandwidth, fidelity, and predictive power. Journal of Personality and Social Psychology, 113(1), 117-143. https://doi.org/10.1037/pspp0000096.

van Roekel, E., Keijsers, L., \& Chung, J. M. (2019). A review of current ambulatory assessment studies in adolescent samples and practical recommendations. Journal of Research on Adolescence, 29(3), 560-577. https://doi.org/10.1111/jora.12471.

Watson, D., Clark, L., \& Tellegen, A. (2007). Development and validation of an internationally reliable short-form of the Positive and Negative Affect Schedule (PANAS). Journal of Cross-Cultural Psychology, 38(2), 227-242. https://doi.org/ 10.1177/0022022106297301.

WHO. (2020). Mental health and psychosocial considerations during the COVID-19 outbreak. Retrieved from https://www.who.int/docs/default-source/coronaviru se/mental-health-considerations.pdf.

Zhou, M., \& Guo, W. (2021). Social factors and worry associated with COVID-19: Evidence from a large survey in China. Social Science and Medicine, 277, Article 113934. https://doi.org/10.1016/j.socscimed.2021.113934. 